Article

\title{
Fourth-Order Adjoint Sensitivity and Uncertainty Analysis of an OECD/NEA Reactor Physics Benchmark: II. Computed Response Uncertainties
}

\author{
Ruixian Fang *(D) and Dan Gabriel Cacuci (D)
}

Citation: Fang, R.; Cacuci, D.G Fourth-Order Adjoint Sensitivity and Uncertainty Analysis of an OECD/NEA Reactor Physics Benchmark: II. Computed Response Uncertainties. J. Nucl. Eng. 2022, 3, 1-16. https://doi.org/10.3390/ jne3010001

Academic Editor: Jeong Ik Lee

Received: 3 December 2021

Accepted: 16 January 2022

Published: 21 January 2022

Publisher's Note: MDPI stays neutral with regard to jurisdictional claims in published maps and institutional affiliations.

Copyright: (C) 2022 by the authors. Licensee MDPI, Basel, Switzerland. This article is an open access article distributed under the terms and conditions of the Creative Commons Attribution (CC BY) license (https:// creativecommons.org/licenses/by/ $4.0 /)$.
Center for Nuclear Science and Energy, Department of Mechanical Engineering, University of South Carolina, Columbia, SC 29208, USA; cacuci@cec.sc.edu

* Correspondence: fangr@cec.sc.edu; Tel.: +1-803-777-7193

\begin{abstract}
This work quantifies the impact of the most important 4th-order sensitivities of the leakage response of a polyethylene-reflected plutonium (PERP) reactor physics benchmark with respect to the benchmark's 180 group-averaged microscopic total cross sections, on the expected value, variance and skewness of the benchmark's leakage response. This work shows that, as the standard deviations of the cross sections increase, the contributions of the 4th-order sensitivities to the response's expected value and variance become significantly larger than the corresponding contributions stemming from the 1st-, 2nd- and 3rd-order sensitivities. Considering a uniform $5 \%$ relative standard deviation for all microscopic total cross sections, the contributions from the 4 th-order sensitivities to the expected value and variance of the PERP leakage response amount to $56 \%$ and 52\%, respectively. Considering $10 \%$ uniform relative standard deviations for the microscopic total cross sections, the contributions from the 4 th-order sensitivities to the expected value increase to nearly $90 \%$. Consequently, if the computed value $L(\boldsymbol{a})$ were considered to represent the actual expected value of the leakage response and the 4th-order sensitivities were neglected, the computed value would represent the actual expected value with an error of $3400 \%$. Furthermore, uniform relative standard deviations of $5 \%$ and larger (10\%) for the microscopic total cross sections cause the higher-order sensitivities to contribute increasingly higher amounts to the response standard deviation: the contributions stemming from the 4th-order sensitivities are larger than the contributions stemming from the 3rd-order sensitivities, which in turn are larger than those stemming from the 2nd-order sensitivities, which are themselves larger than the contributions stemming from the 1st-order sensitivities. This finding evidently underscores the need for computing sensitivities of order higher than first-order. The results obtained in this work also indicate that the 4 th-order sensitivities produce a positive response skewness, causing the leakage response distribution to be skewed towards the positive direction from its expected value. Increasing the parameter standard deviations tends to decrease the value of the response skewness, causing the leakage response distribution to become more symmetrical about the mean value. The results presented in this work highlight the finding that the microscopic total cross section for hydrogen $(\mathrm{H})$ in the lowest ("thermal") energy group is the single most important parameter among the 180 microscopic total cross sections of the PERP benchmark, as it contributes most to the various response moments.
\end{abstract}

Keywords: 1st-order uncertainty; 2nd-order uncertainty; 3rd-order uncertainty; 4th-order uncertainty; higher order uncertainty analysis; total leakage response; microscopic total cross sections; response moments

\section{Introduction}

The previous works [1,2] on 2nd-order and 3rd-order uncertainty analysis for a polyethylene-reflected plutonium (PERP) OECD/NEA reactor physics benchmark [3] revealed that the uncertainties stemming from the 3rd-order sensitivities of the benchmark's leakage response with respect to the total cross sections are significantly larger than 
the uncertainties stemming from the 2nd-order sensitivities, which, in turn, were larger than the uncertainties stemming from the 1st-order sensitivities. These results indicate that a Taylor series expansion of the leakage response up to 3rd-order sensitivities to the total cross sections may be an inadequately accurate representation of the leakage response distribution, which implies that the contributions stemming from 4th-order sensitivities would also need to be quantified in order to assess their relative importance in contributing to the overall uncertainties induced in the leakage response. These 4th-order contributions have now been quantified and their impact on the expectation, variance and skewness of the PERP benchmark's leakage response are reported in this work.

The accompanying work [4], which is designated as Part I, has reported the exact computation of the most important 4th-order sensitivities of the PERP benchmark's total leakage response with respect to the benchmark's 180 group-averaged microscopic total cross sections. The 4th-order sensitivities obtained in [4] are used in this work to compute the contributions of the 4 th-order terms, and compare their impact on the leakage response's expected value, variance and skewness with the corresponding impact stemming from the 1st-, 2nd- and 3rd-order sensitivities.

This work is organized as follows: Section 2 presents the various expressions used for the 4th-order uncertainty analysis of the leakage response for the PERP benchmark. Section 3 presents the numerical results for the 4th-order uncertainty analysis and compares the 4th-order sensitivity contributions with the contributions from the corresponding 1st-, 2nd- and 3rd-order sensitivities. Section 4 discusses the key findings obtained in this work.

\section{Expressions Used in the Fourth-Order Uncertainty Analysis of the PERP Leakage Response}

The expressions for computing the expectation, variance and skewness of a response, up to and including the 4th-order sensitivities, are provided in Appendix A. The first six moments of the unknown multivariate distribution $p_{t}(\mathbf{t})$ of the microscopic total cross sections (which are the model parameters considered in this work) are used in these expressions; these moments are formally defined as follows:

1. The expected value of a model parameter $t_{j 1}$, denoted as $t_{j 1}^{0}$, is defined as follows:

$$
t_{j 1}^{0} \triangleq\left\langle t_{j 1}\right\rangle_{t} \triangleq \int_{D_{t}} t_{j 1} p_{t}(\mathbf{t}) d \mathbf{t}, \quad j 1=1, \ldots, J T X .
$$

The expected values $t_{j 1}^{0}$ are considered to be the components of the following vector of mean (expected) values:

$$
\mathbf{t}^{0} \triangleq\left(t_{1}^{0}, \ldots, t_{J T X}^{0}\right)^{+}
$$

2. The covariance, $\operatorname{cov}\left(t_{j 1}, t_{j 2}\right)$, of two parameters, $t_{j 1}$ and $t_{j 2}$, is defined as follows:

$$
\mu_{2}\left(t_{j 1}, t_{j 2}\right) \triangleq \operatorname{cov}\left(t_{j 1}, t_{j 2}\right) \triangleq\left\langle\left(t_{j 1}-t_{j 1}^{0}\right)\left(t_{j 2}-t_{j 2}^{0}\right)\right\rangle_{t^{\prime}} \quad j 1, j 2=1, \ldots, J T X .
$$

The variance, $\operatorname{var}\left(t_{j 1}\right)$, of a parameter $t_{j 1}$, is defined as follows:

$$
\operatorname{var}\left(t_{j 1}\right) \triangleq\left\langle\left(t_{j 1}-t_{j 1}^{0}\right)^{2}\right\rangle_{t}, \quad j 1=1, \ldots, J T X .
$$

The standard deviation, $\sigma_{j 1}$, of parameter $t_{j 1}$, is defined as follows: $\sigma_{j 1} \triangleq \sqrt{\operatorname{var}\left(t_{j 1}\right)}$; the correlation, $\rho_{j 1 j 2}$, between two parameters $t_{j 1}$ and $t_{j 2}$, is defined as follows:

$$
\rho_{j 1 j 2} \triangleq \operatorname{cov}\left(t_{j 1}, t_{j 2}\right) /\left(\sigma_{j 1} \sigma_{j 2}\right), \quad j 1, j 2=1, \ldots, J T X .
$$


3. The third-order moment (skewness), $\mu_{3}\left(t_{j 1}, t_{j 2}, t_{j 3}\right)$, and the associated third-order correlation, $\tau_{j 1 j 2 j 3}$, among three parameters are defined as follows:

$$
\mu_{3}\left(t_{j 1}, t_{j 2}, t_{j 3}\right) \triangleq\left\langle\left(t_{j 1}-t_{j 1}^{0}\right)\left(t_{j 2}-t_{j 2}^{0}\right)\left(t_{j 3}-t_{j 3}^{0}\right)\right\rangle_{t} \triangleq \tau_{j 1 j 2 j 3} \sigma_{j 1} \sigma_{j 2} \sigma_{j 3}, \quad j 1, j 2, j 3=1, \ldots, J T X .
$$

4. The fourth-order moment (kurtosis), $\mu_{4}\left(t_{j 1}, t_{j 2}, t_{j 3}, t_{j 4}\right)$, and the associated fourthorder correlation, $q_{j 1 j 2 j 3 j 4}$, among four parameters, are defined as follows:

$$
\begin{gathered}
\mu_{4}\left(t_{j 1}, t_{j 2}, t_{j 3}, t_{j 4}\right) \triangleq\left\langle\left(t_{j 1}-t_{j 1}^{0}\right)\left(t_{j 2}-t_{j 2}^{0}\right)\left(t_{j 3}-t_{j 3}^{0}\right)\left(t_{j 4}-t_{j 4}^{0}\right)\right\rangle_{t} \\
\triangleq q_{j 1 j 2 j 3 j 4} \sigma_{j 1} \sigma_{j 2} \sigma_{j 3} \sigma_{j 4}, \quad j 1, j 2, j 3, j 4=1, \ldots, J T X .
\end{gathered}
$$

5. The fifth-order moment, $\mu_{5}\left(t_{j 1}, t_{j 2}, t_{j 3}, t_{j 4}, t_{j 5}\right)$, and the associated fifth-order correlation, $p_{j 1 j 2 j 3 j 4 j 5}$, among five parameters are defined as follows:

$$
\begin{aligned}
& \mu_{5}\left(t_{j 1}, t_{j 2}, t_{j 3}, t_{j 4}, t_{j 5}\right) \triangleq\left\langle\left(t_{j 1}-t_{j 1}^{0}\right)\left(t_{j 2}-t_{j 2}^{0}\right)\left(t_{j 3}-t_{j 3}^{0}\right)\left(t_{j 4}-t_{j 4}^{0}\right)\left(t_{j 5}-t_{j 5}^{0}\right)\right\rangle_{t} \\
& \triangleq p_{j 1 j 2 j 3 j 4 j 5} \sigma_{j 1} \sigma_{j 2} \sigma_{j 3} \sigma_{j 4} \sigma_{j 5}, j 1, j 2, j 3, j 4, j 5=1, \ldots, J T X .
\end{aligned}
$$

6. The sixth-order parameter moment, $\mu_{6}\left(t_{j 1}, t_{j 2}, t_{j 3}, t_{j 4}, t_{j 5}, t_{j 6}\right)$, and the associated sixthorder correlation, $s_{j 1 j 2 j 3 j 4 j 5 j 6}$, among six parameters are defined as follows:

$$
\begin{aligned}
& \mu_{6}\left(t_{j 1}, t_{j 2}, t_{j 3}, t_{j 4}, t_{j 5}, t_{j 6}\right) \\
& \triangleq\left\langle\left(t_{j 1}-t_{j 1}^{0}\right)\left(t_{j 2}-t_{j 2}^{0}\right)\left(t_{j 3}-t_{j 3}^{0}\right)\left(t_{j 4}-t_{j 4}^{0}\right)\left(t_{j 5}-t_{j 5}^{0}\right)\left(t_{j 6}-t_{j 6}^{0}\right)\right\rangle_{t} \\
& \triangleq s_{j 1 j 2 j 3 j 4 j 5 j 6} \sigma_{j 1} \sigma_{j 2} \sigma_{j 3} \sigma_{j 4} \sigma_{j 5} \sigma_{j 6}, j 1, j 2, j 3, j 4, j 5, j 6=1, \ldots, J T X .
\end{aligned}
$$

Only the mean (nominal) values are actually known for the group-averaged microscopic total cross sections involved in the computation of the PERP benchmark leakage response. As has been discussed in [1,2] for the 2nd-order and 3rd-order uncertainty analyses of the leakage response of the PERP benchmark, the second-order moments (i.e., the standard deviations and correlations) for the group-averaged microscopic total cross sections are unavailable for this benchmark. Therefore, the effects on the uncertainties in the leakage response which would stem from the actual correlations among the groupaveraged microscopic total cross sections cannot be exactly quantified [1,2]. When only nominal (mean values) and assumed standard deviations are available, the maximum entropy principle ensures that the least biased distribution that can be assumed for the respective parameters (in the present case: the group-averaged microscopic total cross sections) is the uncorrelated multivariate normal distribution. For uncorrelated normally distributed parameters, the following relations hold:

$$
\begin{gathered}
q_{j 1 j 2 j 3 j 4}=\rho_{j 1 j 2} \rho_{j 3 j 4}+\rho_{j 1 j 3} \rho_{j 2 j 4}+\rho_{j 1 j 4} \rho_{j 2 j 3}, \\
\mu_{2}\left(t_{j 1}, t_{j 2}\right)=\delta_{j 1 j 2} \rho_{j 1 j 2} \sigma_{j 1} \sigma_{j 2}=\sigma_{j 1}{ }^{2}, \\
\mu_{3}\left(t_{j 1}, t_{j 2}, t_{j 3}\right)=0, \\
\mu_{4}\left(t_{j 1}, t_{j 2}, t_{j 3}, t_{j 4}\right)=\left(\delta_{j 1 j 2} \delta_{j 3 j 4}+\delta_{j 1 j 3} \delta_{j 2 j 4}+\delta_{j 1 j 4} \delta_{j 2 j 3}\right) \sigma_{j 1} \sigma_{j 2} \sigma_{j 3} \sigma_{j 4}, \\
\mu_{5}\left(t_{j 1}, t_{j 2}, t_{j 3}, t_{j 4}, t_{j 5}\right)=0, \\
\mu_{6}\left(t_{j 1}, t_{j 2}, t_{j 3}, t_{j 4}, t_{j 5}, t_{j 6}\right)=15 \sigma_{j 1}, i f t_{j 1}=t_{j 2}=t_{j 3}=t_{j 4}=t_{j 5}=t_{j 6} .
\end{gathered}
$$

For uncorrelated and normally-distributed microscopic total cross sections, the expected value of the leakage response takes the following particular expression:

$$
[E(L)]_{t}^{(U, N)}=L\left(\boldsymbol{\alpha}^{0}\right)+[E(L)]_{t}^{(2, U, N)}+[E(L)]_{t}^{(3, U, N)}+[E(L)]_{t}^{(4, U, N)}
$$


where the superscript " $\mathrm{U}, \mathrm{N}$ " indicates contributions from uncorrelated and normallydistributed parameters, the subscript $t$ indicates group-averaged microscopic "total" cross section. In Equation (16), the quantity $L\left(\boldsymbol{\alpha}^{0}\right)$ represents the leakage response computed using the expected cross section values, and $[E(L)]_{t}^{(2, U, N)},[E(L)]_{t}^{(3, U, N)}$ and $[E(L)]_{t}^{(4, U, N)}$ are the contributions from the 2nd-order, 3rd-order and 4th-order response sensitivities, respectively, which are defined as follows:

$$
\begin{gathered}
{[E(L)]_{t}^{(2, U, N)}=\frac{1}{2} \sum_{j 1=1}^{J T X} \frac{\partial^{2} L(\boldsymbol{\alpha})}{\partial^{2} t_{j 1}} \sigma_{j 1}{ }^{2}, J T X=G \times I=180 ;} \\
{[E(L)]_{t}^{(3, U, N)}=\frac{1}{6} \sum_{j 1=1}^{J T X} \sum_{j 2=1}^{J T X} \sum_{j 3=1}^{J T X} \frac{\partial^{3} L(\boldsymbol{\alpha})}{\partial t_{j 1} \partial t_{j 2} \partial t_{j 3}} \mu_{3}\left(t_{j 1}, t_{j 2}, t_{j 3}\right)=0, J T X=G \times I=180 ;} \\
{[E(L)]_{t}^{(4, U, N)}=\frac{1}{4 !} \sum_{j 1=1}^{J T X} \sum_{j 2=1}^{J T X} \sum_{j 3=1}^{J T X} \sum_{j 4=1}^{J T X} \frac{\partial^{4} L(\boldsymbol{\alpha})}{\partial t_{j 1} t_{j 2} \partial t_{j 3} \partial t_{j 4}} \mu_{4}\left(t_{j 1}, t_{j 2}, t_{j 3}, t_{j 4}\right), J T X=G \times I=180,}
\end{gathered}
$$

where $J T X=G \times I=180$ denotes the total number of microscopic total cross sections for $G=30$ groups and $I=6$ isotopes contained in the PERP benchmark [4].

In particular, when only the contributions from the un-mixed 4th-order sensitivities are considered, Equation (19) reduces to the following expression:

$$
[E(L)]_{t}^{(4, U, N)}=\frac{1}{8} \sum_{j 1=1}^{J T X} \frac{\partial^{4} L(\boldsymbol{\alpha})}{\partial^{4} t_{j 1}} \sigma_{j 1}{ }^{4}, J T X=G \times I=180 .
$$

When the group-averaged microscopic total cross sections are uncorrelated and normally-distributed, the expected value of the leakage response does not depend on the 3rd-order and any other odd-order sensitivities.

When the group-averaged microscopic total cross sections are uncorrelated and normally-distributed, the variance of the leakage response for the PERP benchmark takes on the following particular form:

$$
[\operatorname{var}(L)]_{t}^{(U, N)}=[\operatorname{var}(L)]_{t}^{(1, U, N)}+[\operatorname{var}(L)]_{t}^{(2, U, N)}+[\operatorname{var}(L)]_{t}^{(3, U, N)}+[\operatorname{var}(L)]_{t}^{(4, U, N)},
$$

where $[\operatorname{var}(L)]_{t}^{(1, U, N)},[\operatorname{var}(L)]_{t}^{(2, U, N)},[\operatorname{var}(L)]_{t}^{(3, U, N)}$ and $[\operatorname{var}(L)]_{t}^{(4, U, N)}$ denote the 1storder through 4 th-order contributions terms to the variance $[\operatorname{var}(L)]_{t}^{(U, N)}$, respectively, which are defined as follows,

$$
\begin{gathered}
{[\operatorname{var}(L)]_{t}^{(1, U, N)} \triangleq \sum_{j 1=1}^{J T X}\left[\frac{\partial L(\boldsymbol{\alpha})}{\partial t_{j 1}}\right]^{2}\left(\sigma_{j 1}\right)^{2}, \quad J T X=G \times I=180 ;} \\
{[\operatorname{var}(L)]_{t}^{(2, U, N)} \triangleq \frac{1}{2} \sum_{j 1=1}^{J T X} \sum_{j 2=1}^{J T X}\left[\frac{\partial^{2} L(\boldsymbol{\alpha})}{\partial t_{j 1} \partial t_{j 2}} \sigma_{j 1} \sigma_{j 2}\right]^{2}, J T X=G \times I=180 ;} \\
{[\operatorname{var}(L)]_{t}^{(3, U, N)}=\sum_{j 1=1}^{J T X} \sum_{j 2=1}^{J T X}\left[\frac{\partial^{3} L(\boldsymbol{\alpha})}{\partial t_{j 1} \partial t_{j 1} \partial t_{j 2}} \frac{\partial L(\boldsymbol{\alpha})}{\partial t_{j 2}}\right] \sigma_{j 1}{ }^{2} \sigma_{j 2}{ }^{2}} \\
+\frac{15}{36} \sum_{j 1=1}^{J T X}\left[\frac{\partial^{3} L(\boldsymbol{\alpha})}{\partial t_{j 1} \partial t_{j 1} \partial t_{j 1}}\right]^{2} \sigma_{j 1}{ }^{6} ; J T X=G \times I=180 ; \\
{[\operatorname{var}(L)]_{t}^{(4, U, N)}=\frac{1}{2} \sum_{j 1=1}^{J T X}\left[\frac{\partial^{4} L(\boldsymbol{\alpha})}{\left(\partial t_{j 1}\right)^{4}} \frac{\partial^{2} L(\boldsymbol{\alpha})}{\left(\partial t_{j 1}\right)^{2}}\right] \sigma_{j 1}{ }^{6}, J T X=G \times I=180 .}
\end{gathered}
$$


When the group-averaged microscopic total cross sections are uncorrelated and normally-distributed, the 3rd-order moment of the leakage response for the PERP benchmark takes on the following particular form:

$$
\left[\mu_{3}(L)\right]_{t}^{(U, N)}=\left[\mu_{3}(L)\right]_{t}^{(1, U, N)}+\left[\mu_{3}(L)\right]_{t}^{(2, U, N)}+\left[\mu_{3}(L)\right]_{t}^{(3, U, N)}+\left[\mu_{3}(L)\right]_{t}^{(4, U, N)},
$$

where $\left[\mu_{3}(L)\right]_{t}^{(1, U, N)},\left[\mu_{3}(L)\right]_{t}^{(2, U, N)},\left[\mu_{3}(L)\right]_{t}^{(3, U, N)}$ and $\left[\mu_{3}(L)\right]_{t}^{(4, U, N)}$ denote the 1st-order through 4 th-order contributions terms to $\left[\mu_{3}(L)\right]_{t}^{(U, N)}$, respectively, which are defined as follows,

$$
\begin{gathered}
{\left[\mu_{3}(L)\right]_{t}^{(1, U, N)} \triangleq \sum_{j 1=1}^{J T X} \sum_{j 2=1}^{J T X} \sum_{j 3=1}^{J T X} \frac{\partial L(\boldsymbol{\alpha})}{\partial t_{j 1}} \frac{\partial L(\boldsymbol{\alpha})}{\partial t_{j 2}} \frac{\partial L(\boldsymbol{\alpha})}{\partial t_{j 3}} \tau_{j 1 j 2 j 3} \sigma_{j 1} \sigma_{j 2} \sigma_{j 3}=0, \quad J T X=180 ;} \\
{\left[\mu_{3}(L)\right]_{t}^{(2, U, N)} \triangleq 3 \sum_{j 1=1}^{J T X} \sum_{j 2=1}^{J T X} \frac{\partial L(\boldsymbol{\alpha})}{\partial t_{j 1}} \frac{\partial L(\boldsymbol{\alpha})}{\partial t_{j 2}} \frac{\partial^{2} L(\boldsymbol{\alpha})}{\partial t_{j 1} \partial t_{j 2}}\left(\sigma_{j 1} \sigma_{j 2}\right)^{2}+\sum_{j 1=1}^{J T X}\left[\frac{\partial^{2} L(\boldsymbol{\alpha})}{\left(\partial t_{j 1}\right)^{2}}\right]^{3} \sigma_{j 1}{ }^{6}, J T X=180 ;} \\
{\left[\mu_{3}(L)\right]_{t}^{(3, U, N)}=6 \sum_{j 1=1}^{J T X} \frac{\partial L(\boldsymbol{\alpha})}{\partial \boldsymbol{\alpha}_{j 1}} \frac{\partial^{2} L(\boldsymbol{\alpha})}{\left(\partial t_{j 1}\right)^{2}} \frac{\partial^{3} L(\boldsymbol{\alpha})}{\left(\partial t_{j 1}\right)^{3}} \sigma_{j 1}^{6}, J T X=180 ;} \\
{\left[\mu_{3}(L)\right]_{t}^{(4, U, N)}=\frac{3}{2} \sum_{j 1=1}^{J T X}\left[\frac{\partial L(\boldsymbol{\alpha})}{\partial t_{j 1}}\right]^{2} \frac{\partial^{4} L(\boldsymbol{\alpha})}{\left(\partial t_{j 1}\right)^{4}} \sigma_{j 1}{ }^{6}, J T X=180 .}
\end{gathered}
$$

The skewness, denoted as $\gamma_{1}(L)$ of a single response $L(\boldsymbol{\alpha})$, which indicates the degree of the distribution's asymmetry with respect to its mean, is defined as follows [2]:

$$
\left[\gamma_{1}(L)\right]_{t}^{(U, N)}=\left[\mu_{3}(L)\right]_{t}^{(U, N)} /\left\{[\operatorname{var}(L)]_{t}^{(U, N)}\right\}^{3 / 2}
$$

\section{Numerical Results for Fourth-Order Uncertainty Analysis of the PERP Leakage Response}

Using Equations (16) -(31), the effects of the fourth-order sensitivities on the response's expectation, variance and skewness can be quantified by considering uniform standard deviations of $1 \%$ (small), 5\% (moderate), and 10\% (large) of the group-averaged microscopic total cross sections, respectively.

\subsection{The Effects of the Fourth-Order Sensitivities on the Response Expectation}

The effects of the fourth-order sensitivities on the expected value of the leakage response are computed using Equations (16)-(20). The results thus obtained are presented in Table 1.

Table 1. Comparison of Expected Values for Various Relative Standard Deviations (RSD) of the Normally-Distributed and Uncorrelated Microscopic Total Cross Sections.

\begin{tabular}{cccc}
\hline Expected Value & RSD = 1\% & RSD $=\mathbf{5 \%}$ & RSD $=\mathbf{1 0} \%$ \\
\hline$L\left(\boldsymbol{\alpha}^{0}\right)$ & $1.765 \times 10^{6}$ & $1.765 \times 10^{6}$ & $1.765 \times 10^{6}$ \\
{$[E(L)]_{t}^{(2, U, N)}$} & $4.598 \times 10^{4}$ & $1.149 \times 10^{6}$ & $4.598 \times 10^{6}$ \\
{$[E(L)]_{t}^{(3, U, N)}$} & 0.0 & 0.0 & 0.0 \\
{$[E(L)]_{t}^{(4, U, N)}$} & $6.026 \times 10^{3}$ & $3.766 \times 10^{6}$ & $6.026 \times 10^{7}$ \\
{$[E(L)]_{t}^{(U, N)}=L\left(\boldsymbol{\alpha}^{0}\right)+\sum_{i=2}^{4}[E(L)]_{t}^{(i, U, N)}$} & $1.817 \times 10^{6}$ & $6.681 \times 10^{6}$ & $6.662 \times 10^{7}$ \\
\hline
\end{tabular}

As shown in Table 1, when considering a small relative standard deviation of $1 \%$ for each of the uncorrelated microscopic total cross sections of the isotopes included in the PERP benchmark, the effects of the 2nd-order and 4th-order sensitivities through 
$[E(L)]_{t}^{(2, U, N)} \approx 2.5 \% \times[E(L)]_{t}^{(U, N)}$ and $[E(L)]_{t}^{(4, U, N)} \approx 0.3 \% \times[E(L)]_{t}^{(U, N)}$ to the expected response value $[E(L)]_{t}^{(U, N)}$ are both negligibly small.

For a moderate relative standard deviation of $5 \%$ for each of the uncorrelated microscopic total cross sections, the results presented in Table 1 show that $[E(L)]_{t}^{(2, U, N)} \approx 65 \% \times$ $L\left(\alpha^{0}\right) \approx 17 \% \times[E(L)]_{t}^{(U, N)}$, indicating the contributions from the 2nd-order sensitivities to the expected response are around $65 \%$ of the computed leakage value $L\left(\alpha^{0}\right)$, and contribute around $17 \%$ to the expected value $[E(L)]_{t}^{(U, N)}$ of the leakage response. By comparison, the results presented in Table 1 show that $[E(L)]_{t}^{(4, U, N)} \approx 213 \% \times L\left(\alpha^{0}\right) \approx 56 \% \times[E(L)]_{t}^{(U, N)}$, indicating that the contributions from the 4th-order sensitivities to the expected response are about 2.1 times larger than the computed leakage value $L\left(\alpha^{0}\right)$, and contribute around 56\% to the expected value $[E(L)]_{t}^{(U, N)}$. Therefore, if the computed value, $L\left(\alpha^{0}\right)$, is considered to be the actual expected value of the leakage response, neglecting 4 th-order sensitivities would be about $210 \%$ in error for $5 \%$ relative standard deviations for uncorrelated total cross sections.

For a large relative standard deviation of $10 \%$ for each of the uncorrelated microscopic total cross sections, the 2nd- and 4th-order sensitivities cause a significantly larger discrepancy between the leakage response's expected value and its computed value. As shown in the last column of Table 1 , the relation $[E(L)]_{t}^{(2, U, N)} \approx 260 \% \times L\left(\boldsymbol{\alpha}^{0}\right) \approx 7 \% \times[E(L)]_{t}^{(U, N)}$ indicates that contributions of the 2 nd-order term are about 2.6 times larger than the computed leakage value $L\left(\boldsymbol{\alpha}^{0}\right)$, and contribute around $7 \%$ to the expected value $[E(L)]_{t}^{(U, N)}$. For the 4th-order term, the results in Table 1 show that $[E(L)]_{t}^{(4, U, N)} \approx 3414 \% \times L\left(\alpha^{0}\right) \approx$ $90 \% \times[E(L)]_{t}^{(U, N)}$, indicating that the contributions from the 4th-order sensitivities to the expected response are around 34 times larger than the computed leakage value $L\left(\boldsymbol{\alpha}^{0}\right)$ and contribute about $90 \%$ to the expected value $[E(L)]_{t}^{(U, N)}$. Thus, for $10 \%$ relative standard deviations for uncorrelated total cross sections, the computed value $L\left(\boldsymbol{\alpha}^{0}\right)$ would be ca. $3400 \%$ in error by comparison to the actual expected value of the leakage, if the 4 th-order sensitivities were neglected.

Among the 180 microscopic total cross sections, it was found that the parameter $\sigma_{t, 6}^{g=30}$, namely, the total cross sections of isotope \#6 (H) for the 30th group (i.e., the lowest energy group) is the most important parameter affecting the expected value of the leakage response. For example, for a relative standard deviation of $5 \%$ for the uncorrelated microscopic total cross sections, the contribution from the parameter $\sigma_{t, 6}^{g=30}$ to the 2nd-order term $[E(L)]_{t}^{(2, U, N)}$ is ca. $82.5 \%$. More notably, this parameter contributes $99.8 \%$ to the 4 th-order term $[E(L)]_{t}^{(4, U, N)}$, which means that the remaining 179 parameters contribute less than $0.2 \%$. The parameter $\sigma_{t, 6}^{g=30}$ contributes most to the expected value because the 2 nd-order unmixed relative sensitivity of the leakage response with respect to this parameter, i.e., $\left[\partial^{2} L(\boldsymbol{\alpha}) /\left(\partial \sigma_{t, 6}^{30}\right)^{2}\right]\left[\left(\sigma_{t, 6}^{30}\right)^{2} / L(\boldsymbol{\alpha})\right]=4.29 \times 10^{2}$, is the largest among all of the 2 nd-order sensitivities, being ca. 2 orders of magnitudes larger than the values of other 2nd-order unmixed relative sensitivities, as was reported in [1]. Furthermore, the 4th-order unmixed relative sensitivity, $\left[\partial^{4} L(\boldsymbol{\alpha}) /\left(\partial \sigma_{t, 6}^{30}\right)^{4}\right]\left[\left(\sigma_{t, 6}^{30}\right)^{4} / L(\boldsymbol{\alpha})\right]=2.72 \times 10^{6}$, is the largest among all of the 4 th-order unmixed sensitivities, being ca. 4 orders of magnitudes larger than the values of other 4 th-order unmixed relative sensitivities [4]. Through Equations (17) and (20), these large sensitivities lead to significant contributions to the 2nd-order and 4 th-order expected values, respectively. Therefore, the total cross section $\sigma_{t, 6}^{g=30}$ is identified as the most important parameter that significantly affects the expected value of the leakage response for the PERP benchmark. 


\subsection{The Effects of the Fourth-Order Sensitivities on the Response's Variance}

The effects of the fourth-order sensitivities on the variance of the leakage response are computed using Equations (21) - (25), considering parameter relative standard deviations of $1 \%, 5 \%$ and $10 \%$, respectively. The results thus obtained are presented in Table 2 .

Table 2. Comparison of Variances for Various Relative Standard Deviations (RSD) of the NormallyDistributed and Uncorrelated Microscopic Total Cross Sections.

\begin{tabular}{cccc}
\hline Variances & RSD = 1\% & RSD = 5\% & RSD = 10\% \\
\hline$[\operatorname{var}(L)]_{t}^{(1, U, N)}$ & $3.419 \times 10^{10}$ & $8.549 \times 10^{11}$ & $3.419 \times 10^{12}$ \\
{$[\operatorname{var}(L)]_{t}^{(2, U, N)}$} & $2.879 \times 10^{9}$ & $1.799 \times 10^{12}$ & $2.879 \times 10^{13}$ \\
{$[\operatorname{var}(L)]_{t}^{(3, U, N)}$} & $9.841 \times 10^{9}$ & $2.338 \times 10^{13}$ & $1.236 \times 10^{15}$ \\
{$[\operatorname{var}(L)]_{t}^{(4, U, N)}$} & $1.825 \times 10^{9}$ & $2.852 \times 10^{13}$ & $1.825 \times 10^{15}$ \\
{$[\operatorname{var}(L)]_{t}^{(U, N)}=\sum_{i=1}^{4}[\operatorname{var}(L)]_{t}^{(i, U, N)}$} & $4.874 \times 10^{10}$ & $5.456 \times 10^{13}$ & $3.093 \times 10^{15}$ \\
\hline
\end{tabular}

For the relative standard deviation of $1 \%$ of the uncorrelated microscopic total cross sections, the results presented in Table 2 indicate that $[\operatorname{var}(L)]_{t}^{(1, U, N)} \approx 70 \% \times[\operatorname{var}(L)]_{t}^{(U, N)}$, $[\operatorname{var}(L)]_{t}^{(2, U, N)} \approx 6 \% \times[\operatorname{var}(L)]_{t}^{(U, N)},[\operatorname{var}(L)]_{t}^{(3, U, N)} \approx 20 \% \times[\operatorname{var}(L)]_{t}^{(U, N)}$, and $[\operatorname{var}(L)]$ ${ }_{t}^{(4, U, N)} \approx 4 \% \times[\operatorname{var}(L)]_{t}^{(U, N)}$. These results indicate that, for very small relative standard deviations (e.g., 1\%), the contributions from the 1st-order sensitivities to the response variance are significantly larger (ca. 70\%) than those from higher order sensitivities. By comparison, the 2nd-order sensitivities contribute about $6 \%$ to the response variance, the 3 rd-order sensitivities contribute about $20 \%$ to the response variance, while the 4 th-order ones only contribute about $4 \%$ to the response variance.

Figure 1 depicts the uncertainties of the leakage response arising solely from the 1st, 2nd-, 3rd- and 4th-order sensitivities, respectively, assuming $1 \%$ uniform relative standard deviation for all of the uncorrelated microscopic total cross sections. In Figure 1, the following quantities are plotted:

(i) the leakage response $L\left(\boldsymbol{\alpha}^{0}\right)$ of the PERP benchmark;

(ii) the expected value $[E(L)]_{t}^{(U, N)}$ of the leakage response;

(iii) the standard deviation, $S D^{(1)}$, for the leakage response arising solely from the 1storder sensitivities;

(iv) the standard deviation, $S D^{(2)}$, for the leakage response arising solely from the 2ndorder sensitivities;

(v) the standard deviation, $S D^{(3)}$, for the leakage response stemming solely from the 3rd-order sensitivities; and

(vi) the standard deviation, $S D^{(4)}$, for the leakage response stemming solely from the 4th-order sensitivities. 


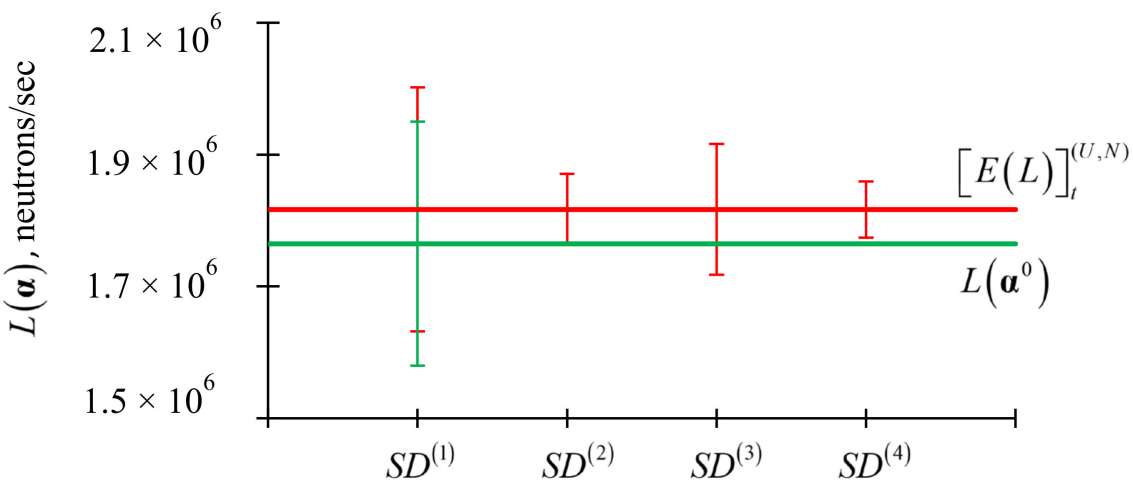

Figure 1. Comparison of $L\left(\boldsymbol{\alpha}^{0}\right) \pm S D^{(1)}$ (in green), $[E(L)]_{t}^{(U, N)} \pm S D^{(1)}, S D^{(2)}, S D^{(3)}, S D^{(4)}$ (in red), due to $1 \%$ standard deviations of the uncorrelated microscopic total cross sections.

In Figure 1, the green-colored plots involve solely 1st-order sensitivities, while the red-colored plots depict the contributions from 1st-, 2nd-, 3rd- and 4th-order sensitivities.

The results presented in Figure 1 show that for small relative standard deviations (e.g., $1 \%$ ) of the uncorrelated microscopic total cross sections, the values of the standard deviations of the leakage response do not decrease monotonically as their respective order increases, but display the following oscillatory pattern: $S D^{(1)} \gg S D^{(3)}>S D^{(2)}>S D^{(4)}$. For a typical relative standard deviation of $5 \%$ for the uncorrelated microscopic total cross sections, the results presented in Table 2 indicate that $[\operatorname{var}(L)]_{t}^{(1, U, N)} \approx 2 \% \times[\operatorname{var}(L)]_{t}^{(U, N)}$, $[\operatorname{var}(L)]_{t}^{(2, U, N)} \approx 3 \% \times[\operatorname{var}(L)]_{t}^{(U, N)},[\operatorname{var}(L)]_{t}^{(3, U, N)} \approx 43 \% \times[\operatorname{var}(L)]_{t}^{(U, N)}$, and $[\operatorname{var}(L)]$ ${ }_{t}^{(4, U, N)} \approx 52 \% \times[\operatorname{var}(L)]_{t}^{(U, N)}$, which means the contributions from the 3rd- and 4thorder sensitivities to the response variance are remarkably larger than those from the 1st- and 2nd-order ones. The effects of the 1st-, 2nd-, 3rd- and 4th-order sensitivities on the quantities $L\left(\alpha^{0}\right) \pm S D^{(1)}$ and $[E(L)]_{t}^{(U, N)} \pm S D^{(1)}, S D^{(2)}, S D^{(3)}, S D^{(4)}$, for $5 \%$ relative standard deviations of the uncorrelated microscopic total cross sections, are illustrated in Figure 2. As shown in the figure, $S D^{(4)}>S D^{(3)} \gg S D^{(2)}>S D^{(1)}$, which indicates that the standard deviations of the leakage response are diverging as the order of sensitivity increases. Hence, neglecting the fourth-order sensitivities would cause a significant error in quantifying the standard deviation of the leakage response for the PERP benchmark.

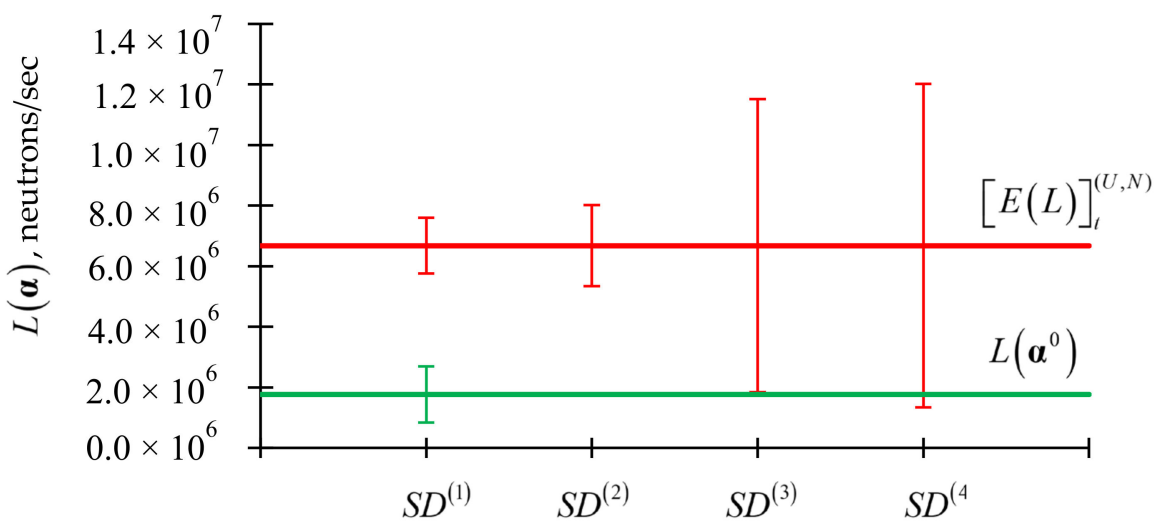

Figure 2. Comparison of $L\left(\boldsymbol{\alpha}^{0}\right) \pm S D^{(1)}$ (in green), $[E(L)]_{t}^{(U, N)} \pm S D^{(1)}, S D^{(2)}, S D^{(3)}, S D^{(4)}$ (in red), due to $5 \%$ standard deviations of the uncorrelated microscopic total cross sections.

For a large relative standard deviation of $10 \%$ of the uncorrelated microscopic total cross sections, the results presented in the last column of Table 2 indicate that $[\operatorname{var}(L)]_{t}^{(1, U, N)} \approx$ $0.1 \% \times[\operatorname{var}(L)]_{t}^{(U, N)},[\operatorname{var}(L)]_{t}^{(2, U, N)} \approx 0.9 \% \times[\operatorname{var}(L)]_{t}^{(U, N)},[\operatorname{var}(L)]_{t}^{(3, U, N)} \approx 40 \% \times$ $[\operatorname{var}(L)]_{t}^{(U, N)}$, and $[\operatorname{var}(L)]_{t}^{(4, U, N)} \approx 59 \% \times[\operatorname{var}(L)]_{t}^{(U, N)}$. Thus, the contributions from the 
3rd- and 4th-order sensitivities amount to ca. $99 \%$ of the total contribution to the response variance while the contributions stemming from the 1st- and 2nd-order sensitivities are negligibly small by comparison. The effects of the 1st-, 2nd-, 3rd- and 4th-order sensitivities on the standard deviations of the leakage response can also be visually seen from the plots for $L\left(\boldsymbol{\alpha}^{0}\right) \pm S D^{(1)}$ and $[E(L)]_{t}^{(U, N)} \pm S D^{(1)}, S D^{(2)}, S D^{(3)}, S D^{(4)}$, for $10 \%$ relative standard deviations of the uncorrelated microscopic total cross sections, as illustrated in Figure 3. Similar to the " $5 \%$ case" above, the plots shown in Figure 3 for the " $10 \%$ case" also present a diverging trend for the standard deviations of the leakage response as the order of sensitivity increases, namely, $S D^{(4)}>S D^{(3)} \gg S D^{(2)}>S D^{(1)}$, but with much larger amplitudes.

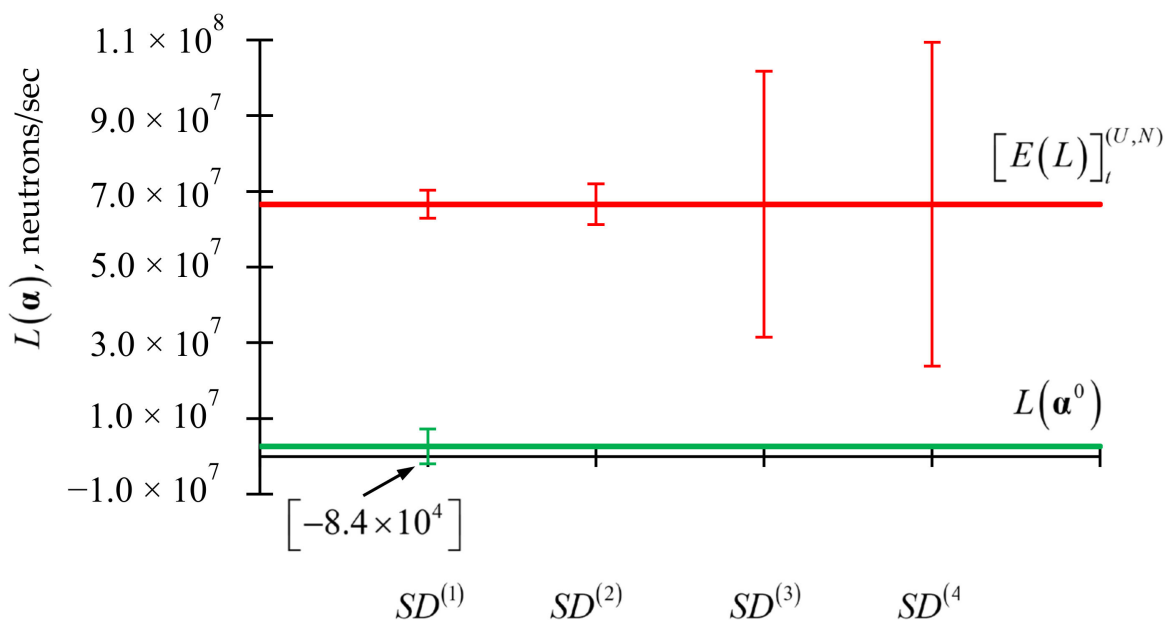

Figure 3. Comparison of $L\left(\boldsymbol{\alpha}^{0}\right) \pm S D^{(1)}$ (in green), $[E(L)]_{t}^{(U, N)} \pm S D^{(1)}, S D^{(2)}, S D^{(3)}, S D^{(4)}$ (in red), due to $10 \%$ standard deviations of the uncorrelated microscopic total cross sections.

The negative value of $L\left(\alpha^{0}\right)-S D^{(1)}$ shown in Figure 3 suggests that $10 \%$ relative standard deviations of the microscopic total cross sections may yield unphysical values for the standard deviation of the response distribution, as was discussed in [2].

The parameter $\sigma_{t, 6}^{g=30}$, namely, the 30th group (i.e., the lowest energy group) of the total cross sections of isotope \#6 $(\mathrm{H})$ contained in the PERP benchmark, was found to provide the largest contributions to the response variance. Specifically, for a relative standard deviation of $5 \%$ for all of the uncorrelated microscopic total cross sections, the contributions from the parameter $\sigma_{t, 6}^{g=30}$ to the various response variance terms $[\operatorname{var}(L)]_{t}^{(1, U, N)},[\operatorname{var}(L)]_{t}^{(2, U, N)}$, $[\operatorname{var}(L)]_{t}^{(3, U, N)},[\operatorname{var}(L)]_{t}^{(4, U, N)}$ and $[\operatorname{var}(L)]_{t}^{(U, N)}$, are $80 \%, 99.8 \%, 99.99 \%, 99.999 \%$ and $99.97 \%$, respectively.

\subsection{The Effects of the Fourth-Order Sensitivities on the 3rd-Order Response Moment and Skewness}

The effects of the fourth-order sensitivities on the 3rd-order moment and skewness of the PERP leakage response are computed using Equations (26)-(31), also considering relative standard deviations of $1 \%, 5 \%$ and $10 \%$, respectively, for all the normally-distributed and uncorrelated microscopic total cross sections. The results are summarized in Table 3. 
Table 3. Comparison of the 3rd-Order Response Moment and Skewness for Various Relative Standard Deviations (RSD) of the Normally-Distributed and Uncorrelated Microscopic Total Cross Sections.

\begin{tabular}{cccc}
\hline 3rd-Order Moment and Skewness & RSD = 1\% & RSD = 5\% & RSD = 10\% \\
\hline$\left[\mu_{3}(L)\right]_{t}^{(1, U, N)}$ & 0 & 0 & 0 \\
{$\left[\mu_{3}(L)\right]_{t}^{(2, U, N)}$} & $6.663 \times 10^{15}$ & $1.070 \times 10^{19}$ & $4.982 \times 10^{20}$ \\
{$\left[\mu_{3}(L)\right]_{t}^{(3, U, N)}$} & $3.948 \times 10^{15}$ & $6.169 \times 10^{19}$ & $3.948 \times 10^{21}$ \\
{$\left[\mu_{3}(L)\right]_{t}^{(4, U, N)}$} & $1.973 \times 10^{15}$ & $3.083 \times 10^{19}$ & $1.973 \times 10^{21}$ \\
{$\left[\mu_{3}(L)\right]_{t}^{(U, N)}=\sum_{i=1}^{4}\left[\mu_{3}(L)\right]_{t}^{(i, U, N)}$} & $1.258 \times 10^{16}$ & $1.032 \times 10^{20}$ & $6.419 \times 10^{21}$ \\
{$\left[\gamma_{1}(L)\right]_{t}^{(U, N)}$} & 1.169 & 0.256 & 0.037
\end{tabular}

Considering 1\% relative standard deviations for the uncorrelated microscopic total cross sections, the results presented in Table 3 indicate that $\left[\mu_{3}(L)\right]_{t}^{(2, U, N)} \approx 53 \% \times$ $\left[\mu_{3}(L)\right]_{t}^{(U, N)},\left[\mu_{3}(L)\right]_{t}^{(3, U, N)} \approx 31 \% \times\left[\mu_{3}(L)\right]_{t}^{(U, N)}$, and $\left[\mu_{3}(L)\right]_{t}^{(4, U, N)} \approx 16 \% \times\left[\mu_{3}(L)\right]_{t}^{(U, N)}$. Thus, for small $(1 \%)$ relative standard deviations, the contributions from the 2nd-order sensitivities to the 3rd-order response moment $\left[\mu_{3}(L)\right]_{t}^{(U, N)}$ are the largest (e.g., around $53 \%$ in this case), followed by the contributions stemming from the 3rd-order sensitivities, while the contributions stemming from the 4th-order sensitivities are the smallest.

Considering 5\% relative standard deviations for the uncorrelated microscopic total cross sections, the results in Table 3 show that $\left[\mu_{3}(L)\right]_{t}^{(2, U, N)} \approx 10 \% \times\left[\mu_{3}(L)\right]_{t}^{(U, N)}$, $\left[\mu_{3}(L)\right]_{t}^{(3, U, N)} \approx 60 \% \times\left[\mu_{3}(L)\right]_{t}^{(U, N)}$, and $\left[\mu_{3}(L)\right]_{t}^{(4, U, N)} \approx 30 \% \times\left[\mu_{3}(L)\right]_{t}^{(U, N)}$. In this case, the contributions from the 3rd-order sensitivities are the largest (e.g., around 60\%), followed by the contributions from the 4 th-order sensitivities, which contribute about 30\%; the smallest contributions stem from the 2nd-order sensitivities.

Considering $10 \%$ relative standard deviations for the uncorrelated microscopic total cross sections, the results in Table 3 indicate that $\left[\mu_{3}(L)\right]_{t}^{(2, U, N)} \approx 8 \% \times\left[\mu_{3}(L)\right]_{t}^{(U, N)}$, $\left[\mu_{3}(L)\right]_{t}^{(3, U, N)} \approx 61 \% \times\left[\mu_{3}(L)\right]_{t}^{(U, N)}$, and $\left[\mu_{3}(L)\right]_{t}^{(4, U, N)} \approx 31 \% \times\left[\mu_{3}(L)\right]_{t}^{(U, N)}$. These results display the same trends as the results for the "RSD $=5 \%$ " case, but the magnitudes of the respective contributions are significantly larger by comparison to the corresponding results for the "RSD $=5 \%$ " case.

For the skewness $\left[\gamma_{1}(L)\right]_{t}^{(\dot{U}, N)}$, the results shown in Table 3 indicate that all the 2nd-, 3rd- and 4th-order sensitivities produce a positive response skewness, which causes the leakage response distribution to be skewed towards the positive direction from its expected value $[E(L)]_{t}^{(U, N)}$. Moreover, the results shown in the last row of Table 3 also indicate that, as the relative standard deviation of the uncorrelated microscopic total cross sections increases from $1 \%$ to $10 \%$, the value of the skewness decreases, thus causing the leakage response distribution to become increasingly more symmetrical about the mean value $[E(L)]_{t}^{(U, N)}$.

In addition, it is noticed that neglecting the 4th-order sensitivities would cause a significant error in the skewness. For example, for the case RSD $=5 \%$, if the 4th-order sensitivities were neglected, the contributions from the 1st-, 2nd- and 3rd-order sensitivities to the skewness would have the value $\left[\gamma_{1}(L)\right]_{t}^{(U, N)}=0.109$, as was reported in [2], which would be $135 \%$ smaller than the more accurate result obtained in this work by including the contributions of the 4 th-order sensitivities.

Similarly, the parameter $\sigma_{t, 6}^{g=30}$ is identified as the most important parameter among the 180 microscopic total cross sections, as it provides the largest contribution to the skewness. For instance, considering the case for RSD $=5 \%$, the contribution stemming from the parameter $\sigma_{t, 6}^{g=30}$ to the skewness $\left[\gamma_{1}(L)\right]_{t}^{(U, N)}$ is $99.991 \%$, which is one order of magnitude larger than the combined contributions from all of other microscopic total cross sections in the PERP benchmark. 


\section{Conclusions}

This work has quantified the impact of the most important 4th-order sensitivities of the leakage response of a polyethylene-reflected plutonium (PERP) reactor physics benchmark with respect to the benchmark's 180 microscopic group-averaged total cross sections, on the response's expected value, variance and skewness. By considering 1\% (small), 5\% (moderate) and 10\% (large) values of the relative standard deviations (RSD) for each of the normally-distributed and uncorrelated microscopic total cross sections, the contributions stemming from the 4th-order sensitivities to the various response moments (e.g., expected value, variance and skewness) were compared with those stemming from the 1st-, 2ndand 3rd-order ones. The following conclusions emerge from this work:

(1) The impact of the 4th-order sensitivities on the expected value of the leakage response varies with the value of the standard deviation of the uncorrelated microscopic total cross sections. Generally, the larger the standard deviations of the microscopic total cross sections, the higher the impact of the 4th-order sensitivities will be on the expected value. For a small relative standard deviation of $1 \%$ for the parameters under consideration, the impact of the 4th-order sensitivities on the expected response value $[E(L)]_{t}^{(U, N)}$ is smaller than the impact of the lower-order sensitivities. However, for a moderate relative standard deviation of $5 \%$, the contributions from the 4 thorder sensitivities are around $56 \%$ of the expected value. When the relative standard deviation is increased to $10 \%$, the contributions from the 4 th-order sensitivities to the expected value increase to nearly $90 \%$. Notably, for the "RSD $=10 \%$ " case, neglecting the 4 th-order sensitivities would cause a large error (ca. 3400\%) if the computed value $L\left(\boldsymbol{\alpha}^{0}\right)$ were considered to be the actual expected value of the leakage response.

(2) The effects of the 4th-order sensitivities on the variance of the leakage response also depends on the value of the standard deviation considered for the microscopic total cross sections. Specifically, if the microscopic total cross sections have small relative standard deviations (e.g., RSD $=1 \%$ ), the 4 th-order sensitivities would only contribute about $4 \%$ to the response variance $[\operatorname{var}(L)]_{t}^{(U, N)}$. For moderate relative standard deviations of $5 \%$, the contributions from the 4 th-order sensitivities increase to $52 \%$. For large relative standard deviations of $10 \%$, the contributions from the 4 th-order sensitivities to the response variance $[\operatorname{var}(L)]_{t}^{(U, N)}$ amounts to $59 \%$, which is significantly larger than the contributions from the corresponding 1st-, 2nd- and 3rd-order sensitivities.

(3) The impact of the 4th-order sensitivities on the standard deviation of the leakage response is as follows: (i) for small relative standard deviations (e.g., 1\%) of the microscopic total cross sections, the uncertainty of the leakage response arising solely from the 1st-order sensitivities are significantly larger than the uncertainties arising solely from the 2nd-, 3rd- and 4th-order sensitivities, respectively, but the following oscillating pattern has been observed: $S D^{(1)} \gg S D^{(3)}>S D^{(2)}>S D^{(4)}$; (ii) when considering moderate and large relative standard deviations (e.g., 5\% and $10 \%$, respectively) for the microscopic total cross sections, the standard deviations of the leakage response appear to diverge as the order of sensitivity increases, i.e., $S D^{(4)}>S D^{(3)} \gg S D^{(2)}>S D^{(1)}$.

(4) The 4th-order sensitivities produce a positive response skewness, causing the leakage response distribution to be skewed towards the positive direction from its expected value. The impact of the 4 th-order sensitivities on the skewness of the leakage response also changes with the value of the standard deviation of the microscopic total cross sections: larger parameter standard deviations tend to decrease the value of the skewness, causing the leakage response distribution to become more symmetrical about the mean value $[E(L)]_{t}^{(U, N)}$.

(5) It was found that, among the 180 microscopic total cross sections, the parameter $\sigma_{t, 6}^{g=30}$ namely, the 30th group (i.e., the lowest energy group) of the total cross sections of isotope \#6 (H) contained in the PERP benchmark, is the single most important 
parameter affecting the PERP benchmark's leakage response, as it has the largest impact on the various response moments. For example, considering that all of the microscopic total cross sections are uncorrelated and have a $5 \%$ relative standard deviation, the $5 \%$ relative standard deviation of $\sigma_{t, 6}^{g=30}$ contributes around $99.8 \%$ to the expected value $[E(L)]_{t}^{(4, U, N)}, 99.97 \%$ to the variance $[\operatorname{var}(L)]_{t}^{(U, N)}$ and $99.99 \%$ to the skewness $\left[\gamma_{1}(L)\right]_{t}^{(U, N)}$.

(6) Because the correlations among the group-averaged microscopic total cross sections are not available for the PERP benchmark under consideration, it was not possible to quantify in this work the impact of the mixed 4th-order sensitivities. As discussed in [2], correlations among the microscopic total cross sections would provide additional contributions to the various response moments (e.g., expected value, variance and skewness).

(7) While the general mathematical expressions presented in this work can be applied to any nuclear reactor system, the numerical results reported in this work are evidently specific to the PERP benchmark.

(8) The general methodology underlying the specific computations of the 4th-order sensitivities used in this work has been presented in [5]. This 4th-order methodology has been recently generalized [6,7] to enable the most efficient computation of exactly obtained mathematical expressions of arbitrarily-high-order (nth-order) sensitivities of a generic system response with respect to all of the parameters (including uncertain domain boundaries) underlying the respective forward/adjoint systems. The mathematical framework underlying this arbitrarily-high order methodology, called the "nth-CASAM-L" methodology, is developed in linearly increasing higher-dimensional Hilbert spaces, as opposed to the exponentially increasing "parameter-dimensional" spaces in which response sensitivities are computed by other methods, thus providing the basis for overcoming the "curse of dimensionality" in sensitivity analysis and all other fields (uncertainty quantification, predictive modeling, etc.) which need such sensitivities. Thus, for the response of a model which comprises a total number of $T P$-parameters - and hence admits TP first-order sensitivities - the 1st-CASAM-L requires 1 additional large-scale adjoint computation (as opposed to at least TP largescale computations, as required by other methods) for computing exactly all of the 1st-order response sensitivities. All of the $T P^{2}$ second-order response sensitivities are computed exactly by the 2nd-CASAM-L in at most TP computations, as opposed to at least $3 T P(T P+1) / 2$ computations required by finite-difference and/or other methods, and so on. For every lower-order sensitivity of interest, the nth-CASAM-L computes the TP "next-higher-order" sensitivities in one adjoint computation performed in a linearly increasing higher-dimensional Hilbert space, thus providing a leap forward in the quest to overcome the "curse of dimensionality" in sensitivity analysis, uncertainty quantification and predictive modeling.

(9) The need for computing higher-order (i.e., higher than first-order) sensitivities (functional derivatives) of model responses with respect to the model parameters has been underscored in $[8,9]$. Using an analytically solvable model of neutron scattering in a hydrogenous medium for which all of the response's relative sensitivities had the same absolute value of unity, it was shown in [9] that the wider the distribution of model parameters, the higher the order of sensitivities needed to achieve a desired level of accuracy in representing the response and in computing the response's expectation, variance, skewness and kurtosis. If only first-order sensitivities are considered, the third-order moment of the response is always zero. Hence, a "first-order sensitivity and uncertainty quantification" will always produce an erroneous third moment (and, hence, skewness) of the predicted response distribution, unless the unknown response distribution happens to be symmetrical. At least second-order sensitivities must be used in order to estimate the third-order moment (and, hence, the skewness) of the response distribution. With pronounced skewness, standard statistical inference procedures such as constructing a confidence interval for the mean (expectation) of 
a computed/predicted model response will be not only incorrect, in the sense that the true coverage level will differ from the nominal (e.g., 95\%) level, but the error probabilities will be unequal on each side of the predicted mean. Thus, the truncation of Taylor expansion of the response (as a function of parameters) depends both on the magnitudes of the response sensitivities to parameters and the parameter uncertainties involved: if the uncertainties are small, then a 4th-order expansion suffices, in most cases, for obtaining relatively accurate results. In any case, the truncation error of a convergent Taylor-series can be quantified a priori. If the parameter uncertainties are large, the Taylor series may diverge, so one would need to consider asymptotic expansions. Of course, if the parameter uncertainties are large, all statistical methods are doomed to produce unreliable results for large-scale, realistic problems, involving many uncertain parameters.

Author Contributions: D.G.C. has conceived and directed the research reported herein, developed the mathematical expressions of the fourth-order comprehensive adjoint sensitivity analysis methodology to compute 4th-order sensitivities of the PERP benchmark's leakage response with respect to the benchmarks' microscopic total cross sections, and the uncertainty equations for response moments. R.F. has performed all of the numerical calculations. Methodology, D.G.C.; Writing-original draft, R.F.; Writing-review \& editing, D.G.C. and R.F. All authors have read and agreed to the published version of the manuscript.

Funding: This research received no external funding.

Institutional Review Board Statement: Not applicable.

Informed Consent Statement: Not applicable.

Data Availability Statement: Data can be available upon request from the authors.

Conflicts of Interest: The authors declare no conflict of interest.

Appendix A. General Expressions for the Expectation, Variance, and Third-Order Response Moment, up to Fourth-Order Response Sensitivities

Appendix A.1. General Expression for the Expectation

Up to 4 th-order response sensitivities, the 1st-order moment, i.e., the expectation $E\left(R_{k}\right)$, of a response $R_{k}$ has the following expression:

$$
\begin{aligned}
& E\left(R_{k}\right)=R_{k}\left(\mathbf{t}^{0}\right)+\frac{1}{2} \sum_{j 1=1}^{T P} \sum_{j 2=1}^{T P} \frac{\partial^{2} R_{k}\left(\mathbf{t}^{0}\right)}{\partial t_{j 1} \partial t_{j 2}} \rho_{j 1 j 2} \sigma_{j 1} \sigma_{j 2}+\frac{1}{6} \sum_{j 1=1}^{T P} \sum_{j 2=1}^{T P} \sum_{j 3=1}^{T P} \frac{\partial^{3} R_{k}\left(\mathbf{t}^{0}\right)}{\partial t_{j 1} \partial t_{j 2} \partial t_{j 3}} \tau_{j 1 j 2 j 3} \sigma_{j 1} \sigma_{j 2} \sigma_{j 3} \\
& +\frac{1}{4 !} \sum_{j 1=1}^{T P} \sum_{j 2=1}^{T P} \sum_{j 3=1}^{T P} \sum_{j 4=1}^{T P} \frac{\partial^{4} R_{k}\left(\mathbf{t}^{0}\right)}{\partial t_{j 1} \partial t_{j 2} \partial t_{j 3} \partial t_{j 4}} q_{j 1 j 2 j 3 j 4} \sigma_{j 1} \sigma_{j 2} \sigma_{j 3} \sigma_{j 4} .
\end{aligned}
$$

\section{Appendix A.2. General Expressions for the Response Variance}

$\mathrm{Up}$ to 4 th-order response sensitivities, the 2 nd-order moment, i.e., the covariance between two responses $R_{k}$ and $R_{l}$ has the following expressions:

The 1st-order sensitivity contribution, $\operatorname{cov}\left(R_{k}^{(1)}, R_{l}^{(1)}\right)$, to the covariance $\operatorname{cov}\left(R_{k}, R_{l}\right)$ is defined as follows:

$$
\operatorname{cov}\left(R_{k}^{(1)}, R_{l}^{(1)}\right)=\sum_{j 1=1}^{T P} \sum_{j 2=1}^{T P} \frac{\partial R_{k}\left(\mathbf{t}^{0}\right)}{\partial t_{j 1}} \frac{\partial R_{l}\left(\mathbf{t}^{0}\right)}{\partial t_{j 2}} \rho_{j 1 j 2} \sigma_{j 1} \sigma_{j 2} .
$$


The 2nd-order sensitivity contribution, $\operatorname{cov}\left(R_{k}^{(2)}, R_{l}^{(2)}\right)$, to the covariance $\operatorname{cov}\left(R_{k}, R_{l}\right)$ is defined as follows:

$$
\begin{gathered}
\operatorname{cov}\left(R_{k}^{(2)}, R_{l}^{(2)}\right)=\frac{1}{2} \sum_{j 1=1}^{T P} \sum_{j 2=1}^{T P} \sum_{j 3=1}^{T P}\left(\frac{\partial^{2} R_{k}\left(\mathbf{t}^{0}\right)}{\partial t_{j 1} \partial t_{j 2}} \frac{\partial R_{l}\left(\mathfrak{t}^{0}\right)}{\partial t_{j 3}}+\frac{\partial R_{k}\left(\mathbf{t}^{0}\right)}{\partial t_{j 1}} \frac{\partial^{2} R_{l}\left(\mathfrak{t}^{0}\right)}{\partial t_{j 2} \partial t_{j 3}}\right) \tau_{j 122 j 3} \sigma_{j 1} \sigma_{j 2} \sigma_{j 3} \\
\quad+\frac{1}{4} \sum_{j 1=1}^{T P} \sum_{j 2=1}^{T P} \sum_{j 3=1}^{T P} \sum_{j 4=1}^{T P} \frac{\partial^{2} R_{k}\left(\mathbf{t}^{0}\right)}{\partial t_{j j} \partial t_{j 2}} \frac{\partial^{2} R_{l}\left(\mathfrak{t}^{0}\right)}{\partial t_{j 3} \partial t t_{j 4}}\left(q_{j 1 j 2 j 3 j 4}-\rho_{j 1 j 2} \rho_{j 3 j 4}\right) \sigma_{j 1} \sigma_{j 2} \sigma_{j 3} \sigma_{j 4} .
\end{gathered}
$$

The 3rd-order sensitivity contribution, $\operatorname{cov}\left(R_{k}^{(3)}, R_{l}^{(3)}\right)$, to the covariance $\operatorname{cov}\left(R_{k}, R_{l}\right)$ is defined as follows:

$$
\begin{aligned}
& \operatorname{cov}\left(R_{k}^{(3)}, R_{l}^{(3)}\right)=\frac{1}{6} \sum_{j 1=1}^{T P} \sum_{j 2=1}^{T P} \sum_{j 3=1}^{T P} \sum_{j 4=1}^{T P}\left(\frac{\partial^{3} R_{k}\left(\mathbf{t}^{0}\right)}{\partial t_{j 1} \partial t_{j} \partial t_{j 3}} \frac{\partial R_{l}\left(\mathbf{t}^{0}\right)}{\partial t_{j 4}}+\frac{\partial R_{k}\left(\mathbf{t}^{0}\right)}{\partial t_{j 1}} \frac{\partial^{3} R_{l}(\mathbf{t})}{\partial t_{j 2} \partial t t_{j} \partial t_{j 4}}\right) q_{j 1 j 2 j 3 j 4} \sigma_{j 1} \sigma_{j 2} \sigma_{j 3} \sigma_{j 4} \\
& +\frac{1}{12} \sum_{j 1=1}^{T P} \sum_{j 2=1}^{T P} \sum_{j 3=1}^{T P} \sum_{j 4=1}^{T P} \sum_{j 5=1}^{T P} \frac{\partial^{3} R_{k}\left(\mathbf{t}^{0}\right)}{\partial t_{j 1} \partial t_{j 2} \partial t t_{j 3}} \frac{\partial^{2} R_{l}\left(\mathbf{t}^{0}\right)}{\partial t_{j 4} \partial t_{j 5}}\left(p_{j 1 j 2 j 3 j 4 j 5}-\tau_{j 1 j 2 j 3} \rho_{j 4 j 5}\right) \sigma_{j 1} \sigma_{j 2} \sigma_{j 3} \sigma_{j 4} \sigma_{j 5} \\
& +\frac{1}{12} \sum_{j 1=1}^{T P} \sum_{j 2=1}^{T P} \sum_{j 3=1}^{T P} \sum_{j 4=1}^{T P} \sum_{j 5=1}^{T P} \frac{\partial^{2} R_{k}\left(\mathbf{t}^{0}\right)}{\partial t_{j 1} \partial t_{j 2}} \frac{\partial^{3} R_{l}\left(\mathbf{t}^{0}\right)}{\partial t_{j 3} \partial t_{j} \partial t_{j 5}}\left(p_{j 1 j 2 j 3 j 4 j 5}-\rho_{j 1 j 2} \tau_{j 3 j 4 j 5}\right) \sigma_{j 1} \sigma_{j 2} \sigma_{j 3} \sigma_{j 4} \sigma_{j 5} \\
& +\frac{1}{36} \sum_{j 1=1}^{T P} \sum_{j 2=1}^{T P} \sum_{j 3=1}^{T P} \sum_{j 4=1}^{T P} \sum_{j 5=1}^{T P} \sum_{j 6=1}^{T P} \frac{\partial^{3} R_{k}\left(\mathfrak{t}^{0}\right)}{\partial t_{j 1} \partial t_{j 2} \partial t_{j 3}} \frac{\partial^{3} R_{l}\left(\mathfrak{t}^{0}\right)}{\partial t_{j 4} \partial t_{j 5} \partial t_{j 6}}\left(s_{j 1 j 2 j 3 j 4 j 5 j 6}-\tau_{j 1 j 2 j 3} \tau_{j 4 j 5 j 6}\right) \sigma_{j 1} \sigma_{j 2} \sigma_{j 3} \sigma_{j 4} \sigma_{j 5} \sigma_{j 6} .
\end{aligned}
$$

The 4 th-order sensitivity contribution, $\operatorname{cov}\left(R_{k}^{(4)}, R_{l}^{(4)}\right)$, to the covariance $\operatorname{cov}\left(R_{k}, R_{l}\right)$ is defined as follows:

$$
\begin{gathered}
\operatorname{cov}\left(R_{k}^{(4)}, R_{l}^{(4)}\right)=\frac{1}{24} \sum_{j 1=1}^{T P} \sum_{j 2=1}^{T P} \sum_{j 3=1}^{T P} \sum_{j 4=1}^{T P} \sum_{j 5=1}^{T P}\left[\frac{\partial^{4} R_{k}\left(\mathbf{t}^{0}\right)}{\partial t_{j 1} \partial t_{j 2} \partial t_{j 3} \partial t_{j 4}} \frac{\partial R_{l}\left(\mathbf{t}^{0}\right)}{\partial t_{j 5}}+\frac{\partial R_{k}\left(\mathbf{t}^{0}\right)}{\partial t_{j 1}} \frac{\partial^{4} R_{l}\left(\mathbf{t}^{0}\right)}{\partial t_{j 2} \partial t_{j 3} \partial t t_{j 4} \partial t_{j 5}}\right] \\
+\frac{1}{48} \sum_{j 1=1}^{T P} \sum_{j 2=1}^{T P} \sum_{j 3=1}^{T P} \sum_{j 4=1}^{T P} \sum_{j 5=1}^{T P} \sum_{j 6=1}^{T P} \frac{\partial^{4} R_{k}\left(\mathbf{t}^{0}\right)}{\partial t_{j 1} \partial t_{j 2} \partial t t_{j 3} \partial t_{j 4} \sigma_{j 1}} \frac{\partial^{2} R_{l}\left(\mathbf{t}^{0}\right)}{\partial t_{j 5} \partial t t_{j 6}}\left(s_{j 1 j 2 j 3 j 4 j 5 j 6}-q_{j 1 j 2 j 3 j 4} \rho_{j 5 j 6}\right) \sigma_{j 1} \sigma_{j 2} \sigma_{j 3} \sigma_{j 4} \sigma_{j 5} \sigma_{j 6} \\
+\frac{1}{48} \sum_{j 1=1}^{T P} \sum_{j 2=1}^{T P} \sum_{j 3=1}^{T P} \sum_{j 4=1}^{T P} \sum_{j 5=1}^{T P} \sum_{j 6=1}^{T P} \frac{\partial^{2} R_{k}\left(\mathbf{t}^{0}\right)}{\partial t_{j 1} \partial t t_{j 2}} \frac{\partial^{4} R_{l}\left(\mathbf{t}^{0}\right)}{\partial t_{j 3} \partial t_{j 4} \partial t_{j 5} \partial t_{j 6}}\left(s_{j 1 j 2 j 3 j 4 j 5 j 6}-\rho_{j 1 j 2} q_{j 3 j 4 j 5 j 6}\right) \sigma_{j 1} \sigma_{j 2} \sigma_{j 3} \sigma_{j 4} \sigma_{j 5} \sigma_{j 6} .
\end{gathered}
$$

Appendix A.3. General Expressions for the Third-Order Response Moment

Up to 4th-order response sensitivities, the 3rd-order moment between three responses $R_{k}, R_{l}$ and $R_{m}$ has the following expressions:

The 1st-order sensitivity contribution, $\mu_{3}^{(1)}\left(R_{k}, R_{l}, R_{m}\right)$, to the 3rd-order moment $\mu_{3}\left(R_{k}, R_{l}, R_{m}\right)$ is defined as follows:

$$
\mu_{3}^{(1)}\left(R_{k}, R_{l}, R_{m}\right)=\sum_{j 1=1}^{T P} \sum_{j 2=1}^{T P} \sum_{j 3=1}^{T P} \frac{\partial R_{k}\left(\mathbf{t}^{0}\right)}{\partial t_{j 1}} \frac{\partial R_{l}\left(\mathbf{t}^{0}\right)}{\partial t_{j 2}} \frac{\partial R_{m}\left(\mathbf{t}^{0}\right)}{\partial t_{j 3}} \tau_{j 1 j 2 j 3} \sigma_{j 1} \sigma_{j 2} \sigma_{j 3} .
$$

The 2nd-order sensitivity contribution, $\mu_{3}^{(2)}\left(R_{k}, R_{l}, R_{m}\right)$, to the 3rd-order moment $\mu_{3}\left(R_{k}, R_{l}, R_{m}\right)$ is defined as follows: 


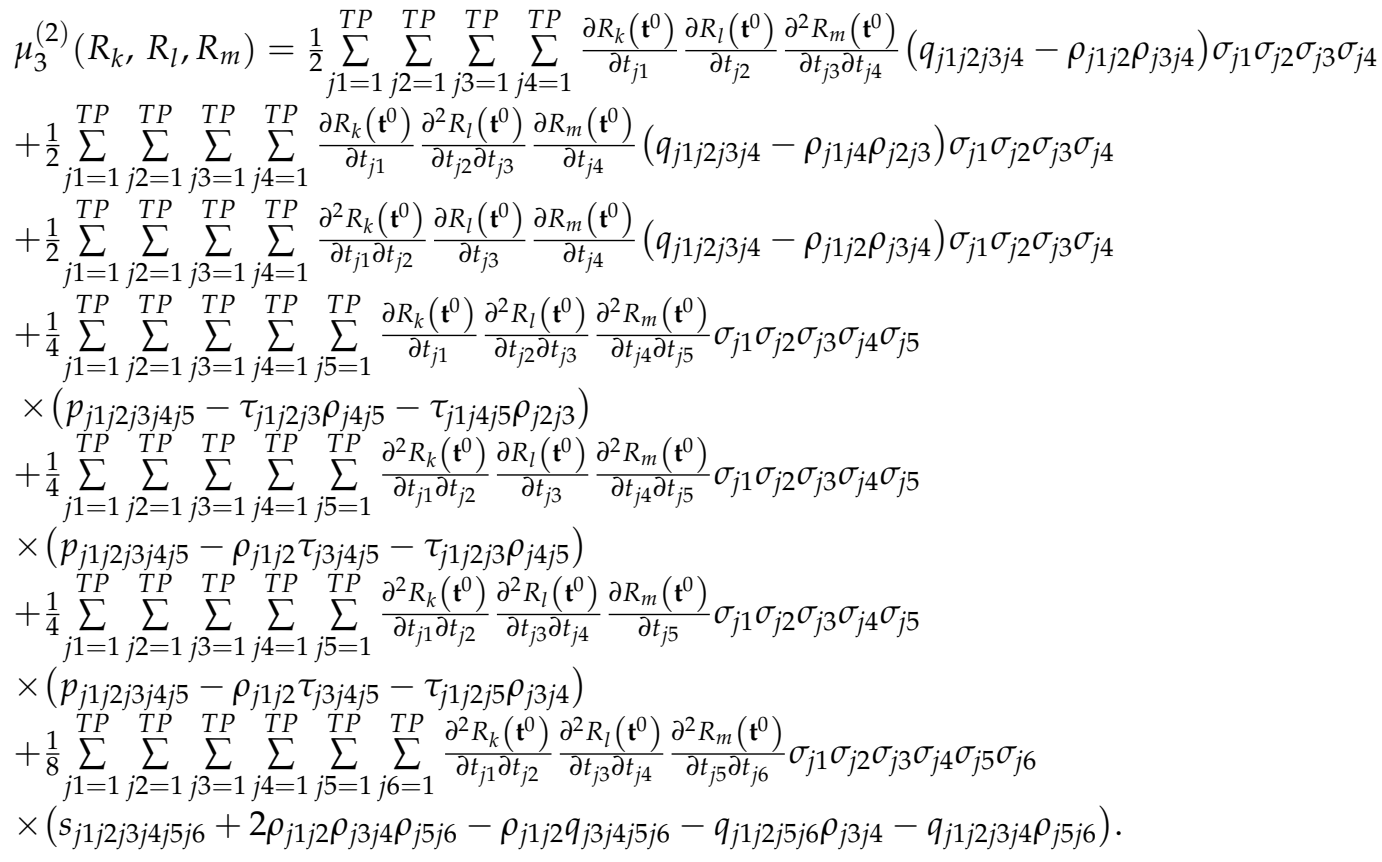

The 3rd-order sensitivity contribution, $\mu_{3}^{(3)}\left(R_{k}, R_{l}, R_{m}\right)$, to the 3 rd-order moment $\mu_{3}\left(R_{k}, R_{l}, R_{m}\right)$ is defined as follows:

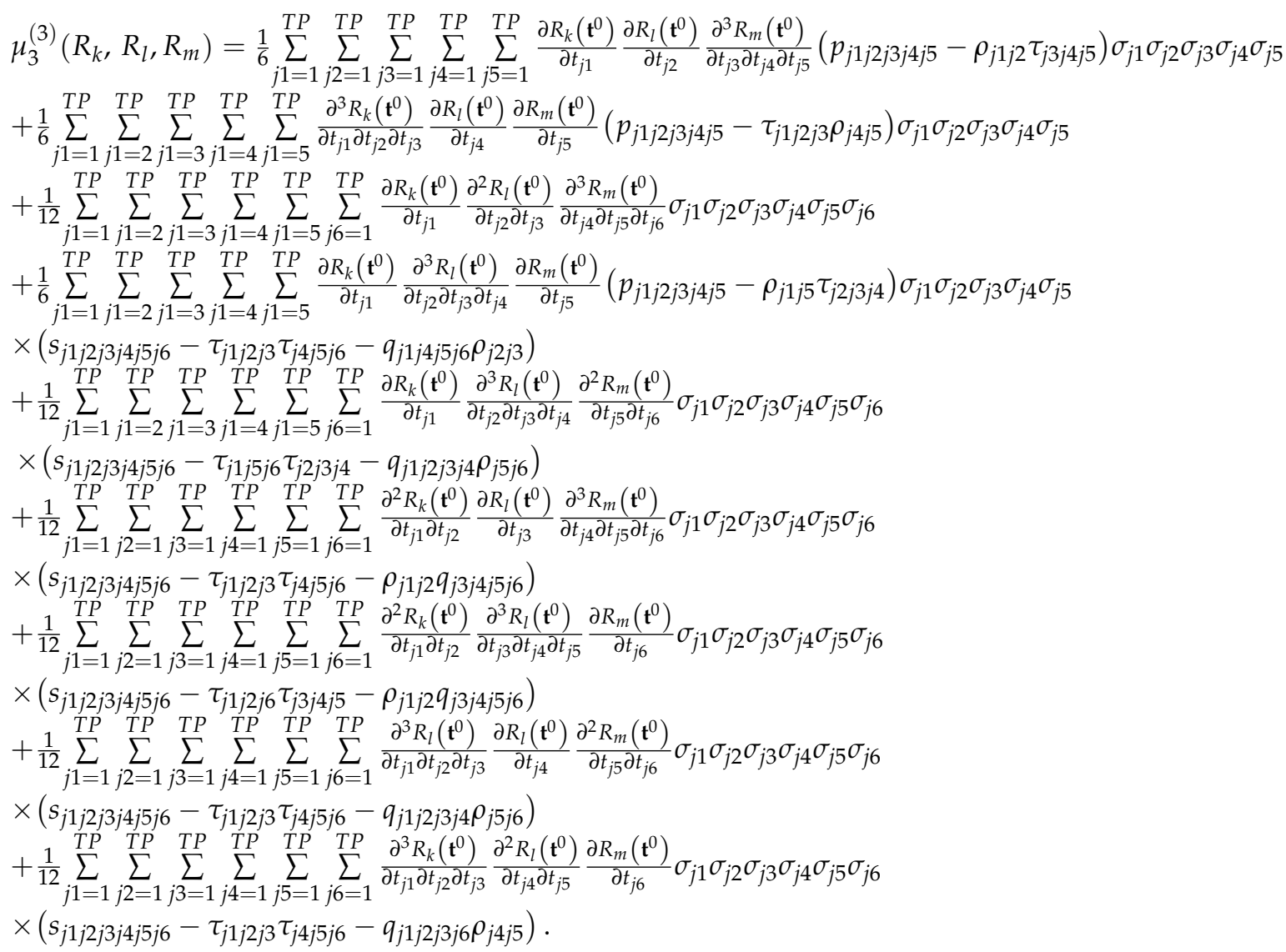


The 4th-order sensitivity contribution, $\mu_{3}^{(4)}\left(R_{k}, R_{l}, R_{m}\right)$, to the 3rd-order moment $\mu_{3}\left(R_{k}, R_{l}, R_{m}\right)$ is defined as follows:

$$
\begin{aligned}
& \mu_{3}^{(4)}\left(R_{k}, R_{l}, R_{m}\right)=\frac{1}{24} \sum_{j 1=1}^{T P} \sum_{j 2=1}^{T P} \sum_{j 3=1}^{T P} \sum_{j 4=1}^{T P} \sum_{j 5=1}^{T P} \sum_{j 6=1}^{T P} \frac{\partial R_{k}\left(\mathbf{t}^{0}\right)}{\partial t_{j 1}} \frac{\partial R_{l}\left(\mathbf{t}^{0}\right)}{\partial t_{j 2}} \frac{\partial^{4} R_{m}\left(\mathbf{t}^{0}\right)}{\partial t_{j 3} \partial t_{j 4} \partial t_{j 5} \partial t_{j 6}} \\
& \times\left(s_{j 1 j 2 j 3 j 4 j 5 j 6}-\rho_{j 1 j 2} q_{j 3 j 4 j 5 j 6}\right) \sigma_{j 1} \sigma_{j 2} \sigma_{j 3} \sigma_{j 4} \sigma_{j 5} \sigma_{j 6} \\
& +\frac{1}{24} \sum_{j 1=1}^{T P} \sum_{j 1=2}^{T P} \sum_{j 1=3}^{T P} \sum_{j 1=4}^{T P} \sum_{j 1=5}^{T P} \sum_{j 6=1}^{T P} \frac{\partial R_{k}\left(\mathbf{t}^{0}\right)}{\partial t_{j 1}} \frac{\partial^{4} R_{l}\left(\mathbf{t}^{0}\right)}{\partial t_{j 2} \partial t_{j 3} \partial t_{j 4} \partial t_{j 5}} \frac{\partial R_{m}\left(\mathbf{t}^{0}\right)}{\partial t_{j 6}} \\
& \quad \times\left(s_{j 1 j 2 j 3 j 4 j 5 j 6}-\rho_{j 1 j 6} q_{j 2 j 3 j 4 j 5}\right) \sigma_{j 1} \sigma_{j 2} \sigma_{j 3} \sigma_{j 4} \sigma_{j 5} \sigma_{j 6} \\
& +\frac{1}{24} \sum_{j 1=1}^{T P} \sum_{j 1=2}^{T P} \sum_{j 1=3}^{T P} \sum_{j 1=4}^{T P} \sum_{j 1=5}^{T P} \sum_{j 6=1}^{T} \frac{\partial^{4} R_{k}\left(\mathbf{t}^{0}\right)}{\partial t_{j 1} \partial t_{j 2} \partial t_{j 3} \partial t_{j 4}} \frac{\partial R_{l}\left(\mathbf{t}^{0}\right)}{\partial t_{j 5}} \frac{\partial R_{m}\left(\mathbf{t}^{0}\right)}{\partial t_{j 6}} \\
& \times\left(s_{j 1 j 2 j 3 j 4 j 5 j 6}-q_{j 1 j 2 j 3 j 4} \rho_{j 5 j 6}\right) \sigma_{j 1} \sigma_{j 2} \sigma_{j 3} \sigma_{j 4} \sigma_{j 5} \sigma_{j 6} .
\end{aligned}
$$

\section{References}

1. Cacuci, D.G.; Fang, R.; Favorite, J.A. Comprehensive Second-Order Adjoint Sensitivity Analysis Methodology (2nd-ASAM) Applied to a Subcritical Experimental Reactor Physics Benchmark: I. Effects of Imprecisely Known Microscopic Total and Capture Cross Sections. Energies 2019, 12, 4219. [CrossRef]

2. Fang, R.; Cacuci, D.G. Third-Order Adjoint Sensitivity Analysis of an OECD/NEA Reactor Physics Benchmark: III. Response Moments. Am. J. Comput. Math. 2020, 10, 559-570. [CrossRef]

3. Valentine, T.E. Polyethylene-reflected plutonium metal sphere subcritical noise measurements, SUB-PU-METMIXED-001. In International Handbook of Evaluated Criticality Safety Benchmark Experiments; NEA/NSC/DOC(95)03/I-IX.; Nuclear Energy Agency: Paris, France, 2006.

4. Fang, R.; Cacuci, D.G. Fourth-Order Adjoint Sensitivity and Uncertainty Analysis of an OECD/NEA Reactor Physics Benchmark: I. Computed Sensitivities. J. Nucl. Eng. 2021, 2, 24. [CrossRef]

5. Cacuci, D.G. Fourth-Order Comprehensive Adjoint Sensitivity Analysis (4th-CASAM) of Response-Coupled Linear Forward/Adjoint Systems. I. Theoretical Framework. Energies 2021, 14, 3335. [CrossRef]

6. Cacuci, D.G. The nth-Order Comprehensive Adjoint Sensitivity Analysis Methodology for Response-Coupled Forward/Adjoint Linear Systems (nth-CASAM-L): I. Mathematical Framework. Energies 2021, 14, 8314. [CrossRef]

7. Cacuci, D.G. The nth-Order Comprehensive Adjoint Sensitivity Analysis Methodology for Response-Coupled Forward/Adjoint Linear Systems (nth-CASAM-L): II. Illustrative Application. Energies 2021, 14, 8315. [CrossRef]

8. Cacuci, D.G. On the Need to Determine Accurately the Impact of Higher-Order Sensitivities on Model Sensitivity Analysis, Uncertainty Quantification and Best-Estimate Predictions. Energies 2021, 14, 6318. [CrossRef]

9. Cacuci, D.G. High-Order Deterministic Sensitivity Analysis and Uncertainty Quantification: Review and New Developments. Energies 2021, 14, 6715. [CrossRef] 